\title{
Distributed Detection in Cognitive Radio Networks
}

\author{
Shweta Kumari, Gunjan Kumari
}

\begin{abstract}
With the increase of wireless services and devices, and the need to maintain their QoS requirements, the demand for spectral bandwidth has increased in recent years. The traditional spectrum allocation techniques have led to inefficient spectrum utilisation. In this project, we analyse a new paradigm called Dynamic Spectrum Access, using energy detection methods in case of real-valued and complex-valued signals. An energy efficient detection is also proposed in a distributed cognitive network for the case of a single $S U$ and $N$ PUs, where $N>1$. The cognitive radio network also has to pay a cost whenever a SU switches a channel. In this scenario, detection algorithms are also proposed absed on myopic approach.
\end{abstract}

Index Terms - Cognitive Radio, Spectrum Sensing, Energy Detector, Distributed Detection.

\section{INTRODUCTION}

Wireless communication systems have seen a tremendous progress in the last few decades. Rapid growth in wireless communication systems, increased the number of users and in-

creased the number of applications like Voice Telephony, Web browsing, etc., and also in-creased the demand for more spectral bandwidth. However, the fixed amount of spectrum

and the growing number of services and users have led to spectrum scarcity. As the spectrum is the most heavily regulated and expensive natural resource around the world, and almost all the spectrum suitable for wireless communications are allocated to rightful licensees, a new way of meeting the demands of the spectrum needs to be explored. Since 2002, a large number of spectrum measurements have been carried out throughout the world for analysing the spectrum utilization globally. From January 2004 to August 2005, the US Federal Communications Commission (FCC) has surveyed the spectrum utilization in frequency bands $30 \mathrm{MHz}-3000 \mathrm{MHz}$ in US, turning out to be just $5.2 \%$ utilisation. This leads to spectrum scarcity. DSA is an efficient technique for solving spectrum scarcity. It is implemented using CR (Cognitive Radio).

\section{A. Objective of Paper}

1) Analysis of Energy Detection methods for real and CSCG primary signals.

2) Algorithm development and simulation of the same for spectrum sensing in a distributed environment in case of

Revised Manuscript Received on December 15, 2019.

Shweta Kumari, Department of Electronics and Communication Engineering, VFSTR Guntur, A.P, India. fsc.kshweta@gmail.com

Gunjan Kumari, Department of Electronics and Communication Engineering IIITDM, Chennai, Tamil Nadu, India raghuvansi072@gmail.com
1 PU and N SUs, where N>1, with energy constraints and channel switching cost.

\section{B. Literature Survey}

Since spectrum is one of the most crucial components, a variety of methods have peen proposed in [1]. A comparative study of schemes in [2] reveal that Energy Detection is the most widely used sensing approach, since it does not need any a priori information about primary transmission characteristics. However, it takes a long sensing time for detecting low signal to noise ratio primary signal. Numerous other approaches are suggested for energy detection in [3-4]. A dynamic estimation of noise power is proposed in [4], the need of which arises from the fact that the performance of ED is limited by the estimation error of noise signal power. In [5] authors describe the concept of adaptive threshold to be used based on signal to interference noise power. It ensures a higher throughput than the previous approach, but the disadvantage is that it leads to a higher false alarm. J.Bazerque et al. [6] proposed that spectrum sensing can be implemented either in a centralized or distributed manner. Also, the sensing may be cooperative or non -cooperative. It is shown that decentralized cooperative sensing utilizes the spatial diversity of SUs more efficiently than the centralized approach and is more robust. A cooperative spectrum sensing is studied in [7] in order to reduce the detection time and improve the robustness. An OFDM based system is proposed in [8] wherein the decisions from all local SUs are taken and given to a center.The centre makes the final decision on the basis of logical combinations. Waled et al. [9] proposed a sequential detection procedure called Sequential Probability Ratio Test (SPRT) in which samples are taken sequentially. The log likelihood ratio for each $\mathrm{SU}$ is computed and compared to two thresholds (upper and lower) in a binary hypothesis testing problem. If LLR falls in between the two thresholds, the detector takes the next sample. The process is repeated until the LLR crosses any of the thresholds. The number of samples in Wald's SPRT has no bounds resulting in its inefficiency to be used practically. In [10] Xin et al. proposed Shift Chi Square Test (SSCT) where the SU stops sensing till a certain number is reached. In another work [11], it is shown that correlation between the sensors severly decrease the cooperative gain. In [12] authors propose a consensus based algorithm The approach here takes into account the collaborative nature of SUs. The previous described works do not take into account the impact of energy constraints on sensing and access policies. Also, very few work are there in the field of a distributed

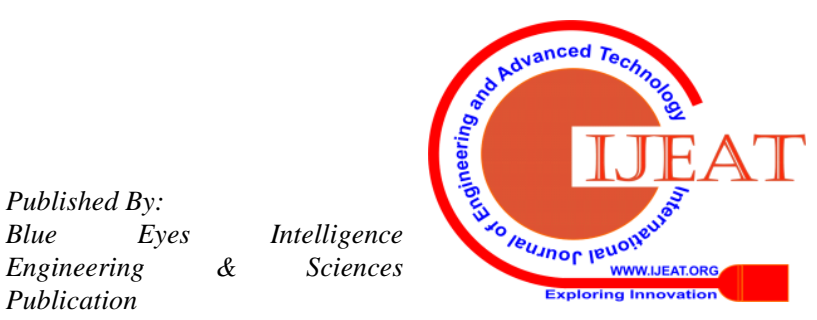


non-cooperative environment.

In this paper, a distributed environment is considered with 1 PUs and $\mathrm{N}$ SUs where $\mathrm{N}>1$. The PUs and SU are independently working without any knowledge of each other's

state. Under the energy constraint, an algorithm is developed and simulated. Also, another algorithm in case the SU wishes to minimize the number of switches in case of a switching cost involved.

\section{ANALYSIS OF ENERGY DETECTOR}

\section{A. System Model}

We consider a CRN comprising a set of $N$ Primary Users and 1 SU. PU intends to use the channel, but when it is not transmitting, SU senses and uses it. Time is slotted, non-negative and indexed by $k$. Suppose we are interested in PU X, where $X \in\{1,2, \ldots N\}$. Since the received signal is discrete, we consider $m$ samples of it. In case of a nonfading channel, the basic hypothesis model for transmitter detection technique in time slot $k$ is:

$$
\begin{array}{lll}
H_{0}: & Y_{k, q}=N_{k, q} & q=1,2, \ldots m \\
H_{1}: & Y_{k, q}=X_{k, q}+N_{k, q} & q=1,2, \ldots m
\end{array}
$$

\section{B. Problem Formulation}

Keeping in view the interests of SU, two probabilities are of interest. The first probability being the Probability of false alarm, $\left(P_{f a}\right)$ and the second being the Probability of detection $\left(P_{d}\right)$. A higher probability of false alarm leads to poor spectrum usage by the SU whereas a higher probability of detection ensures better chances of SU not interfering with the PU. The two probabilities are worked out.

\section{Analysis}

Assumption 1: $X \sim N\left(0, \sigma_{x}^{2}\right)$ and $N \sim N\left(0, \sigma_{n}^{2}\right)$

$$
\begin{aligned}
& P_{f a}=\frac{\sqrt{m}}{\sigma_{n}^{2}} Q\left(\sqrt{m}\left(\frac{t-1}{\sqrt{2} \sigma_{n}^{2}}\right)\right) . \\
& P_{d}=\frac{1}{\sqrt{2}} Q\left(\sqrt{\left.\frac{m}{2}\left(\frac{t-(\gamma+1) \sigma_{n}^{2}}{(\gamma+1) \sigma_{n}^{2}}\right)\right)}\right.
\end{aligned}
$$

$$
\text { Assumption } \quad 2 \text { : } \quad X \sim C N\left(0, \sigma_{x}^{2}\right) \quad \text { and }
$$

$$
\begin{gathered}
N \sim C N\left(0, \sigma_{n}^{2}\right) \\
P_{f a}=\frac{1}{\sigma_{0}} Q\left(\sqrt{m}\left(\frac{t}{\sigma_{n}^{2}}-1\right)\right) \\
P_{d}=Q\left(\frac{1}{\sigma_{1}}\left(t-\mu_{1}\right)\right)
\end{gathered}
$$

\section{Simulation}

A CRN of $1 \mathrm{SU}$ and $1 \mathrm{PU}$. The simulation result is for $m=1000$ samples per time slot. The total time slot is 1000. The input signal is real valued and CSCG with mean 0 and variance 1 in Fig. 1 and Fig. 2 respectively. The noise is modelled as Gaussian and CSCG with mean 0 and variance 1 in Fig. 1 and Fig. 2 respectively.

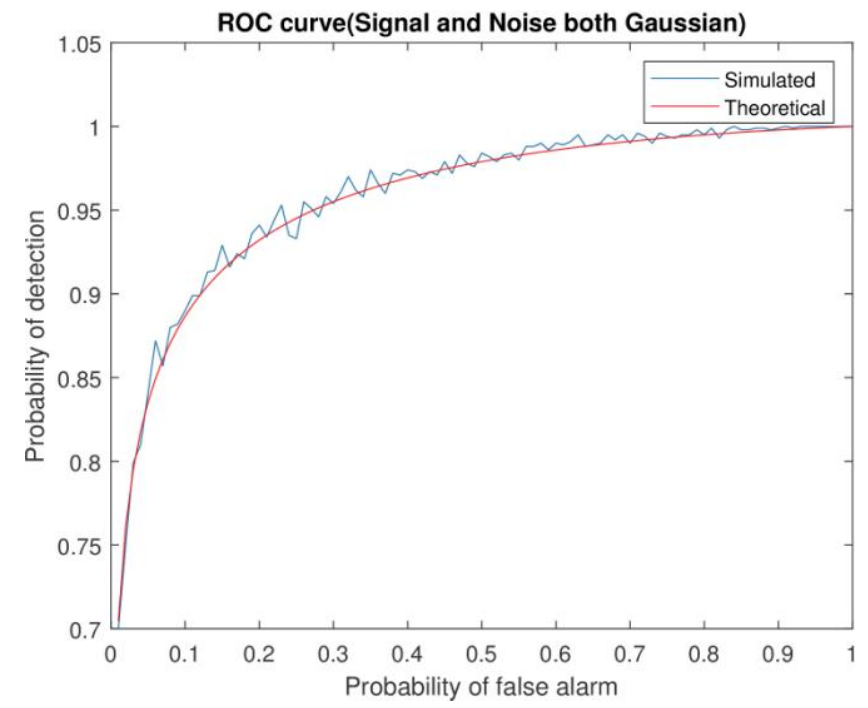

Fig. 1: $P_{d}$ vs $P_{f a}$

ROC curve in case of both input signal and noise signal being real- valued gaussian and in no fading.

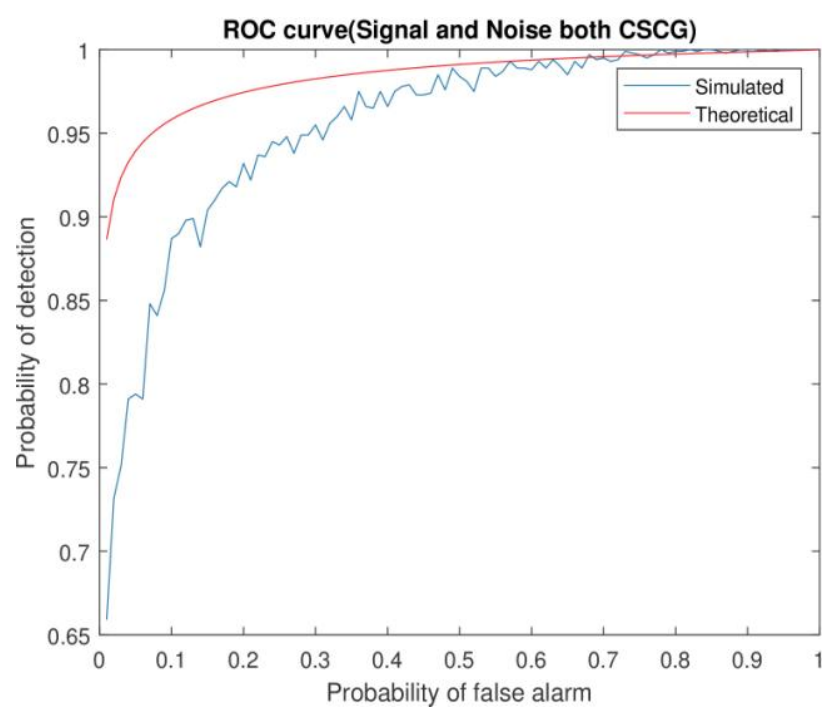

Fig. 2 : $P_{d}$ vs $P_{f a}$

As can be seen from Fig.1 and Fig.2, a trade-off between the two probabilities is required for an efficient working of CRN.

\section{ENERGY EFFICIENT DETECTION}

\section{A. System Model}

We consider a CRN comprising a set of $N$ Primary Users and 1 SU. PU intends to use the channel, but when it is not transmitting, SU senses and uses it. Time is slotted, nonnegative and indexed by $k$. The SU has to sense a channel and make the decision whether to transmit or not. The state of channel $i$, in time slot $k$ can be either busy (0) or idle (1) which is denoted by a theta vector $\theta_{i, k} \in\{0,1\}$.

Blue Eyes Intelligence

\& Sciences

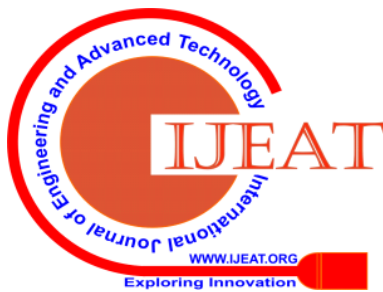


It is assumed that theta for a channel $i$, in a given time slot $k$ is Markov chain and is independent of theta vector for other channels in same time slot.

The state vector of $N$ PUs is therefore, $\Theta_{k}=\left\{\theta_{1, k}, \theta_{2, k}, . . \theta_{N, k}\right\}$. The transition probabilities of the theta vector is given as : $P_{i, k}=\left[p_{00} p_{01} ; p_{10} p_{11}\right]$. Also, the sensing model is assumed as follows:

$$
\begin{aligned}
& P\left(Y_{i, k}=0 \mid \theta_{i, k}=0\right)=\alpha \\
& P\left(Y_{i, k}=0 \mid \theta_{i, k}=1\right)=\beta \\
& P\left(Y_{i, k}=0 \mid \theta_{i, k}=1\right)=1-\alpha \\
& P\left(Y_{i, k}=1 \mid \theta_{i, k}=1\right)=1-\beta
\end{aligned}
$$

\section{B. Problem Formulation}

Since a SU can not make the observations directly, it has to sense the observations. On the basis of these sensing outcomes, SU decides whether to access the channel or not. Since the SU senses channel at every $k$ interval, it may or may not switch to another channel for transmission. If it switches to another channel, i.e. switch to another frequency which means in wireless incurring a cost in terms of delay and packet loss. Also, a limitation on the hardware prevents SU to sense every channel in a given time slot. At a certain interval of time, observations of only a single PU may be available. The SU therefore, needs an efficient channel sensing and access policy to avoid the frequent channel switching unless necessary while keeping in view the limitations on its sensing abilities.

\section{Solution}

Let us consider $\lambda$ as the cost incurred in case of switching to a channel other than the current channel $c$ (suppose). At the beginning of time slot $k$, the SU sees defined state of the system $Y_{k}=\left[\Theta_{k}, c\right]$. Let us introduce a new notation, $\pi_{i, k}$ which denotes the conditional probability of channel $i$ being sensed as idle given past states of the channel, i.e $\pi_{i, k}=P\left(\theta_{i, k}=1 \mid Y_{1}, Y_{2}, . . Y_{k}\right)$. This $\pi_{i, k}$ in time slot $k$ can then be used to generate $\pi$ values for future time slot. The $\pi_{i, k}$ value is calculated as:

For sensed

channel:

$\pi_{i, k+1}=\frac{\left(p_{11} \pi_{i, k}+p_{01}\left(1-\pi_{i, k}\right)\right) \beta}{\left(p_{11} \pi_{i, k}+p_{01}\left(1-\pi_{i, k}\right)\right) \beta+\left(p_{10} \pi_{i, k}+p_{00}\left(1-\pi_{i, k}\right)\right)}$

when $Y_{i, k}=0$

$\pi_{i, k+1}=\frac{\left(p_{11} \pi_{i, k}+p_{01}\left(1-\pi_{i, k}\right)\right)(1-\beta)}{\left(p_{11} \pi_{i, k}+p_{01}\left(1-\pi_{i, k}\right)\right)(1-\beta)+\left(p_{10} \pi_{i, k}+p_{00}\left(1-\pi_{i, k}\right)\right)(}$

When $Y_{i, k}=1$

For unsensed channel:

$\pi_{i, k+1}=p_{00} \pi_{i, k}+p_{01}\left(1-\pi_{i, k}\right)$

\section{Proposed Algorithm}

1. Input vector- $s_{k}=[1,2, \ldots N]$. Output- SU accesses any of the above channel based on sensing policy.

2. for do $k=1: T$

$$
\begin{aligned}
& \text { for do } \mathrm{j}=1: N \\
& \text { Calculate } \max \left(\pi_{j, k}\right) .
\end{aligned}
$$

end for

end for.

3. Select $\max \left(\pi_{j, 1}\right)$ and set $c=j$.

4. for do $k=2: T$

Calculate $\left|\max \left(\pi_{j, k}\right)-\pi_{c, k}\right|$.

5. if $\left|\max \left(\pi_{j, k}\right)-\pi_{c, k}\right|<\mathrm{thr}$

Continue access of channel $c$. else

Switch to channel $j$.

6. end if

7. end for

\section{E. Empirical Study}

A CRN of $1 \mathrm{SU}$ and $N$ PUs. The total number of time slots is $10^{4}$. The threshold values thr varies from 0.1-0.9. A graph of throughput of SU against probability of collision values is plotted. The sensing parameters $\alpha$ and $\beta$ are taken as 0.1 and 0.9 respectively. Mathematically, throughput is defined as:

Throughput $=($ Total No. Of Access/Total time slot $) *(1-$ Probability of collision)

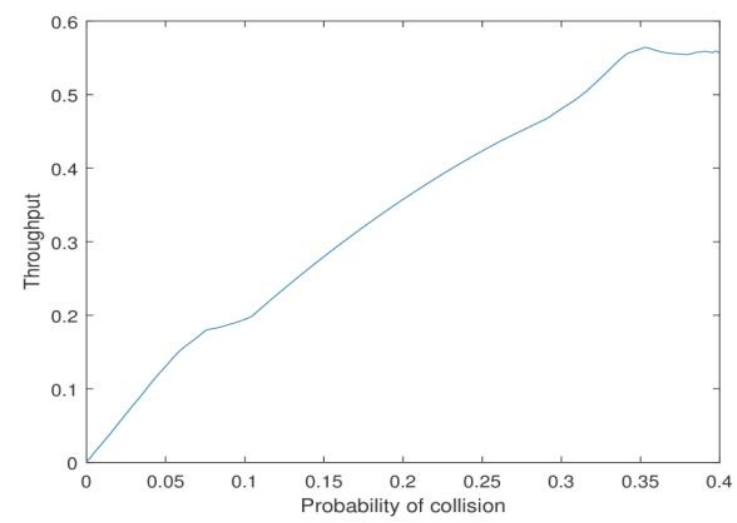

Fig. 3 : Variation of Throughput of SU

with the probability of collision. An almost linearly increasing plot achieves a near flat curve around values $0.38-0.4$.

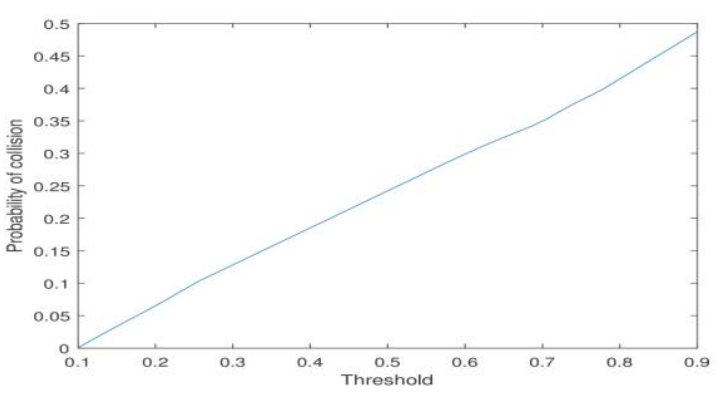

Fig 4: Probability of collision versus Threshold

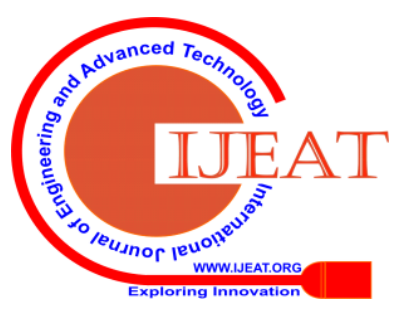


A linearly increasing plot signifies the probability of collision is directly proportional to threshold values.

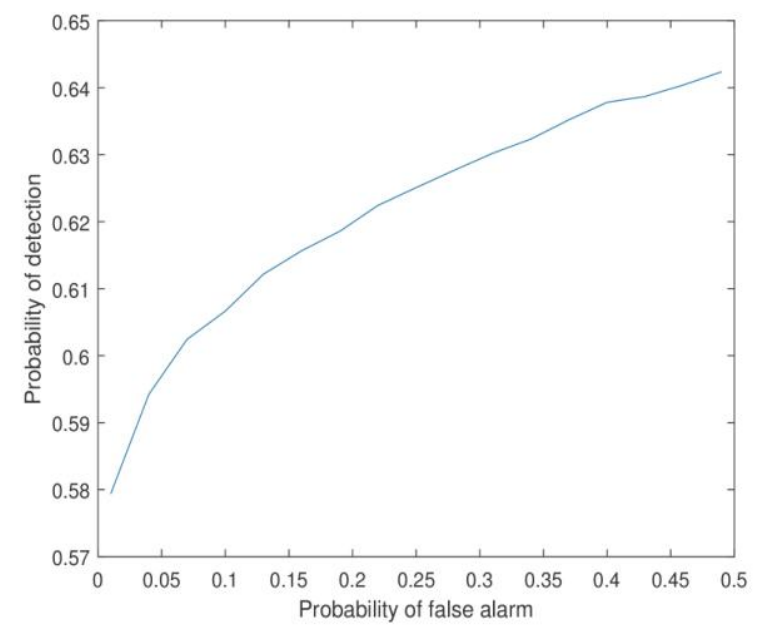

Fig. 5: ROC curve for the proposed algorithm. A steep slope occurs initially followed by a flat region around values $0.45-0.5$

\section{CONCLUSION}

The traditional spectrum allocation techniques have led to inefficient spectrum utilisation. In this project, we analyse a new paradigm called Dynamic Spectrum Access, using energy detection methods in case of real-valued and complex-valued signals. An energy efficient detection is also proposed in a distributed cognitive network for the case of a single SU and $\mathrm{N}$ PUs, where $\mathrm{N}>1$. The cognitive radio network also has to pay a cost whenever a SU switches a channel. In this scenario, detection algorithms are also proposed absed on myopic approach.

\section{REFERENCES}

1. E. Axell, G.Leus, and E.G. Larsson," Spectrum Sensing for Cognitive Radio: state-of-the-art and recent advances," IEEE Signal Processing Mag., vol.29, no.3, pp. 101116, 2012.

2. R. Umar, and A.U.H. Sheikh," A comparative study of spectrum awareness techniques for cognitive radio oriented wireless networks," Physical Communications J. Special Issue:Cognitive Radios, July 2012.

3. S. Gong, P. Wang, and W. Liu, "Spectrum Sensing under distribution uncertainty in cognitive radio networks," IEEE International Conference, Comm. , pp. 1512-1516, 2012.

4. A. Khan , M.H. Rehmani , and M. Reisslein ," Cognitive Radio for Smart Grids: Survey of architectures , spectrum sensing mechanisms, and networking protocols , " IEEE Comm. Surv. Tutorials, vol. 18, no. 1 , pp. 860-898, 2016.

5. M.R. Manesh, N. Kaabouch, and H. Reyes, “ A Bayesian Approach to Estimate and Model SINR in Wireless Networks," International Journal of Communication Systems, Wiley, pp. 1-11, 2016.

6. J. Bazerque and G. Giannakis, "Distributed spectrum sensing for cognitive radio networks by exploiting sparsity,” IEEE Trans. Signal Process., vol. 58, no. 3, pp. 1847-1862, Mar. 2010.

7. G. Ganesan, Y. Li, "Cooperative spectrum sensing in cognitive radio networks", IEEE International Symposium on New Frontiers in Dynamic Spectrum Access Networks, pp. 137-143. 